\title{
Expert System Design for Determining the Harmonic Resonance and the Capacitor Safety Level
}

\author{
Ö. Gül, and R.T. Othan \\ Department of Electrical Engineering \\ Istanbul Technical University \\ Campus of Ayazaga - Maslak, 34469 Istanbul (Turkey) \\ Phone/Fax number:+90 212 2856709, e-mail: gulomer@itu.edu.tr, tuygun85@gmail.com
}

\begin{abstract}
This paper presents a computer based expert system aiming the determination of harmonic resonance and the capacitor safety level. Developed applications for the determination of harmonic resonance are basically depending on the selection of a right harmonic impedance measurement method and the changes observed in the equivalent system impedance due to the occurrence of harmonic resonance. However, developed applications for the determination of capacitor safety level are depending on the international standards which introduce the related indices and limits for the capacitors and the fuzzy logic method. All developed applications were programmed using LabVIEWTM and the data required, is obtained continuously from real-time measurements.
\end{abstract}

\section{Key words}

Expert system, harmonic resonance, capacitor safety, realtime measurements, fuzzy logic.

\section{Introduction}

In electrical circuits, resonance occurs as the inductive reactance becomes equal to the capacitive reactance. The type of the resonance depends on the allocation of inductances and capacitors existing in the circuit. For the electrical power systems, transformers are a good example for system components that have inductive impedance characteristics and also, capacitors positioned to the customer's point of common coupling are a good example for system components that have capacitive impedance characteristics.

Since all of the customers own much or less of the loads that are sources of harmonics, resonance phenomenon becomes much more severe. First and general method for the determination of the resonance conditions of a system is obtaining the harmonic dependent models of the system components. As the conditions of the resonance may differ between phases, three-phase models should be preferred for a correct analysis.

As if the operation conditions of a system component (capacitors in this research e.g.) is also interested, then adequate power flow algorithm (both for the harmonic frequencies and the network type) should be used together with the obtained models.

However, in practical, resonance analysis depending on modelling has some specific difficulties and impossibilities. First of all, as the results of the analysis depend on the component models, accuracy of the results is directly related to the conformity of models to the reality. Secondly, obtaining the three-phase models of all system components are not easy (three-phase transformer models differ due to the connection type of the primary and secondary windings e.g.). In an established plant, estimating the exact line and cable lengths or the differentiation according to the environmental and furnishing conditions may not be possible all the time and finally nowadays, as the electrical power systems have very dynamical structures; variations can't be reflected to the models in an exact way.

Thus, another method is proposed in this research which is mainly based on real-time measurements. According to the method, voltage and current signals are obtained from field measurements. Then the equivalent system impedance $\left(Z_{\mathrm{eq}}\right.$. $)$ is calculated by the help of these measured signals. A possible resonance will be observed from the frequency scan analyse of $\mathrm{Z}_{\mathrm{eq}}$.

With the existence of harmonic components and the occurrence of resonance, the threats caused for the system components are another severe issue. Especially, as the reactance of the capacitors decreases by the increase in frequency, effects of harmonics and the resonance may be more destructive. Possible problems to be seen for the capacitors are over voltages, over currents and over loading.

IEEE 1036-1992 [1] and IEC 60831-1 [2] both define the safe operation indices and limits for the capacitors. Benefiting these standards, fuzzy logic based applications is developed to identify the safety level of capacitors.

\section{Harmonic Resonance}

Two different types of resonance occur in electrical systems. These are series and parallel resonances. 


\section{A. Series Resonance}

When the system equivalent inductance and the capacitance are serially connected to each other, series resonance occurs in a certain frequency. In a series RLC circuit, the equivalent system impedance is given by:

$$
\text { Zeq. }=\mathrm{R}+\mathrm{j}(\mathrm{XL}-\mathrm{XC})
$$

As seen from the equation above, the equivalent system impedance will only consists of the resistance value for the frequency that resonance occurs. By other means, for the resonant frequency, the system impedance has its minimum value and the voltage applied to the ends of the series RLC circuit is in the same phase with the current flowing through.

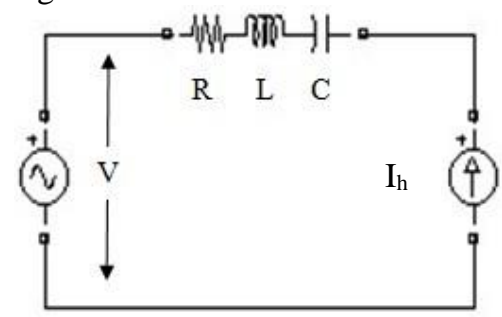

Fig. 1. Series RLC circuit.

In fig. 1, a series RLC circuit is shown. For $\mathrm{R}=1 \Omega$, $\mathrm{L}=633.26 \mathrm{mH}$ and $\mathrm{C}=1 \mu \mathrm{F}$, the equivalent circuit impedance is calculated at different frequencies. The variations obtained in the amplitude and the phase angle of the Zeq. are seen in fig. 2 and fig. 3 respectively.

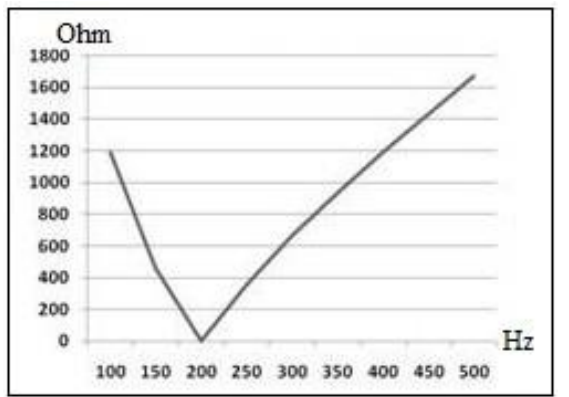

Fig. 2. Changes in the amplitude of the Zeq. in the series RLC circuit.

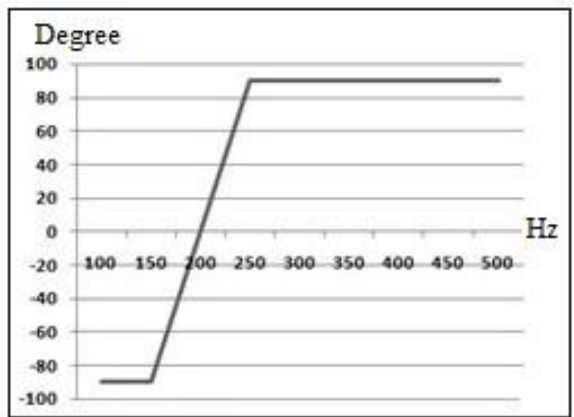

Fig. 3. Changes in the phase angle of the Zeq. in the series RLC circuit.

For the given parameters, a series resonance occurs at 200 $\mathrm{Hz}$ and at $200 \mathrm{~Hz}$, Zeq. gets its minimum value as $1 \Omega$, which is the value of the $\mathrm{R}$ and the phase angles of Zeq. change its sign from negative to positive, after the resonant frequency.

\section{B. Parallel Resonance}

When the system equivalent inductance and the capacitance are connected in parallel to each other, parallel resonance occurs in a certain frequency. Different ways of parallel connection is possible with $\mathrm{R}$, $\mathrm{L}$ and C. In figure 4 , a simple parallel RLC circuit is shown. The equivalent system admittance and impedance of such a circuit is given by:

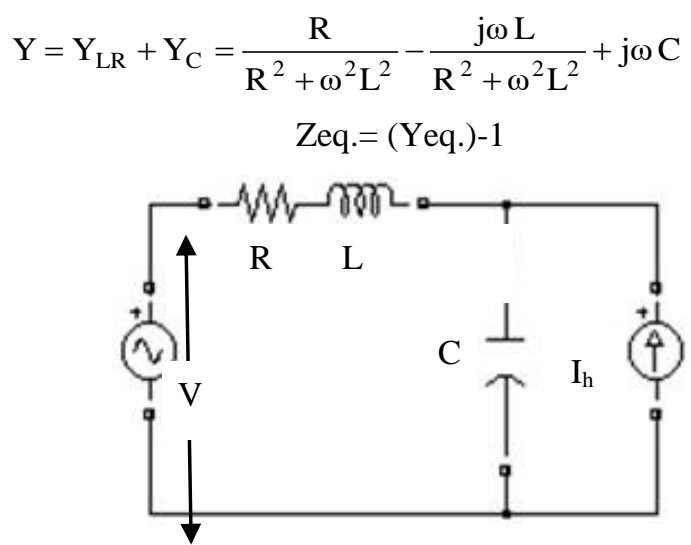

Fig. 4. Parallel RL-C circuit.

In a parallel RL-C circuit as shown in fig. 4 , for $\mathrm{R}=1 \Omega$, $\mathrm{L}=56.29 \mathrm{mH}$ and $\mathrm{C}=5 \mu \mathrm{F}$, the equivalent circuit impedance is calculated at different frequencies. The changes obtained in the amplitude and the phase angle of the Zeq. are seen in fig. 5 and fig. 6 respectively.

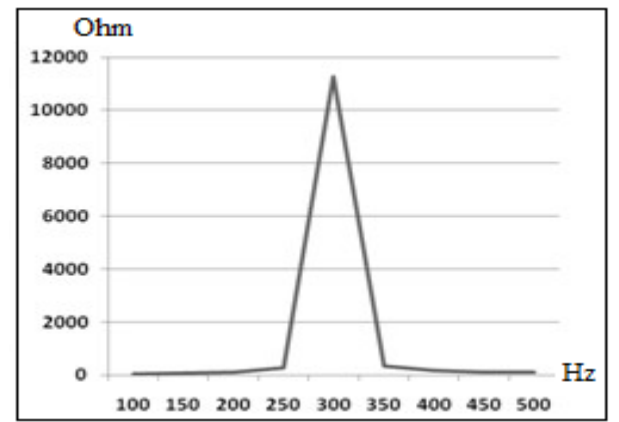

Fig. 5. Changes in the amplitude of the Zeq. in the parallel RL-C circuit.

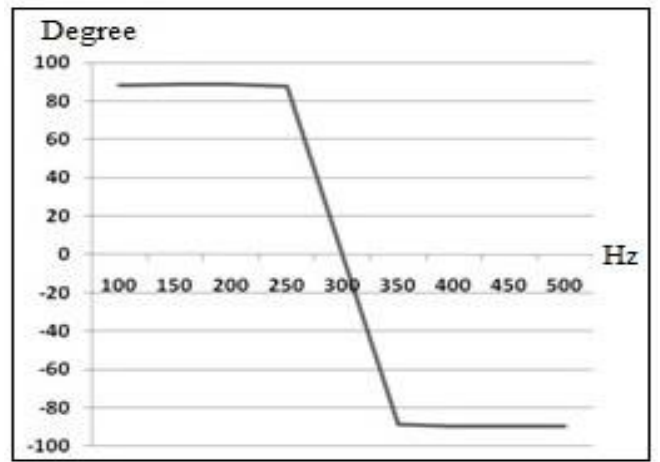

Fig. 6. Changes in the phase angle of the Zeq. in the parallel RL-C circuit.

For given parameters, parallel resonance occurs at 300 $\mathrm{Hz}$ and at $300 \mathrm{~Hz}$, Zeq. gets its maximum value and the phase angles change its sign from positive to negative, after the resonant frequency. 


\section{Harmonic Impedance Measurement Methods}

There is variety of harmonic impedance measurement methods in the literature. Each method has its specific advantages and disadvantages in terms of cost, simplicity and implementation. Generally, the methods can be gathered in two main titles as invasive and non-invasive methods according to the external effect done by the researcher. In figure 7 , these methods are schematized.

Most common examples for an external effect are capacitor switching operations, injection of harmonic components by connection of a power electronic device and switching transformers. In reference [3-5], a capacitor bank is switched on to cause an instantaneous short circuit that result with a current signal in a wide frequency range. In reference [6] a transformer is switched on. Switching a transformer may cause it to reach saturation. Transformer energization can generate high transient currents depending on the remanence and the switching moment. These currents, also known as transient inrush currents, have wide frequency spectrum content with considerable magnitudes. In reference [7] a power electronic converter is used to inject a controlled current spike in a system. Since the transients' strength depends on the switching moment, controlling their magnitudes and suitably disturbing the system for measurement purposes are possible with the control of the firing angle.

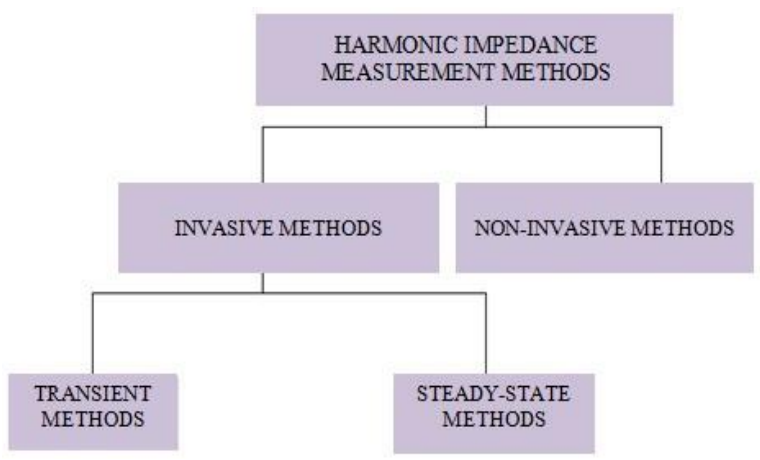

Fig. 7. Harmonic impedance measurement methods.

Invasive methods may divided into two as some researchers use transient signals obtained, after an external effect is applied; while some others use the steady-state signals of a system before and after the external effect is applied.

Non-invasive methods use natural variations of harmonic voltages and currents formed by the harmonic sources operating in the system. In reference [8, 9], non-invasive methods are used to determine harmonic impedances. Most of the researchers that especially desire to determine the harmonic impedance in a wide frequency prefer invasive methods as it gives opportunity to decide the spectral content of the distortive signal. However for this research, it is better to use non-invasive methods as the main purpose is to determine the occurrence of a possible resonance. As known, the occurrence of resonance may be mentioned if there is a distortive signal in the resonant frequency. Otherwise, occurrence of resonance is impossible although the equivalent inductive reactance and the capacitive reactance become equal to each other at a certain frequency.

\section{Capacitor Safety}

\section{A. Effects of Harmonic Resonance on Capacitors}

Harmonic resonance may result with over voltages, over currents and over loading which will damage the system components. Especially capacitors get much more sensitive to the effects of resonance, as their reactance decreases by frequency increment. The definition of the problem and its influence depends on the type of the resonance occurs and the amplitude of the harmonic components, especially at the resonant frequency.

\section{1) Over currents}

True RMS value of the current flowing through capacitor's branch will be much higher with the existence of harmonic components. During series resonance, as the equivalent system impedance gets its minimum value, current component at resonant frequency may become dangerous in means of capacitor safety.

Also during parallel resonance, the continuously circulating current between the system inductance and the capacitance may be several hundreds of times greater than the supply current due to the quality factor at resonance [10].

\section{2) Over voltages}

With the existence of harmonic components, the true RMS value of the voltages calibrated will be greater. Especially during parallel resonance, according to the current component injected at resonant frequency, the voltage values may become dangerous in means of capacitor safety.

During series resonance, the voltages calibrated at the ends of the inductance and the capacitors may be much greater than the supply voltage if the quality factor at resonance is big [10]. For this reason, quality factor is often called as the circuit magnification factor [10].

\section{3) Over loading}

As power is the function of the voltage and the current of the capacitor, the value calculated over these, may reach up to dangerous values. Over loading may exist both in parallel and series resonances according to the system parameters and the magnitude of the harmonic current components injected.

\section{B. Capacitor Safety Indices and Limits}

Capacitor safety indices and limit values are defined by several standards. In Table 1, standard limitations of IEEE 1036-1192 is shown for the apparent power (S), RMS voltage (Vrms) and the RMS current (Irms) of the capacitor [1].

Table I. capacitor loading indices and limits according to ieee std. No. 1036-1992

\begin{tabular}{|l|l|l|}
\hline Index & Description & Limit \\
\hline $\mathrm{S}$ & $\begin{array}{l}\text { Apparent power of the } \\
\text { capacitor(=Irms*Vrms) }\end{array}$ & $135 \%$ \\
\hline Vrms & RMS voltage of the capacitor & $110 \%$ \\
\hline Irms & RMS current of the capacitor & $180 \%$ \\
\hline
\end{tabular}


Another standard that defines the capacitor safety indices and limits is IEC 60831-1. Limitations belonging to this standard are shown in Table 2. RMS voltage of the capacitor is defined as time dependent [2].

Table II. Capacitor loading indices and limits according to iec 60831-1

\begin{tabular}{|c|c|c|c|}
\hline Index & Description & \multicolumn{2}{|l|}{ Limit } \\
\hline Irms & $\begin{array}{l}\text { RMS current } \\
\text { of the } \\
\text { capacitor }\end{array}$ & \multicolumn{2}{|l|}{$130 \%$} \\
\hline \multirow{6}{*}{ Vrms } & \multirow{6}{*}{$\begin{array}{l}\text { RMS voltage } \\
\text { of the } \\
\text { capacitor }\end{array}$} & $\begin{array}{l}\text { Maximum Accepted } \\
\text { Time }\end{array}$ & Limit \\
\hline & & Continuous & $100 \%$ \\
\hline & & $8 \mathrm{~h}$. in every $24 \mathrm{~h}$. & $110 \%$ \\
\hline & & $30 \mathrm{~min}$. In every $24 \mathrm{~h}$. & $115 \%$ \\
\hline & & $5 \mathrm{~min}$. & $120 \%$ \\
\hline & & $1 \mathrm{~min}$. & $130 \%$ \\
\hline
\end{tabular}

\section{Design of the Expert System}

Operating principle of the expert system is based on the determination of harmonic resonance by observing the specific variations in the harmonic impedances and the classification of capacitor operation in terms of safety using fuzzy logic. Flow chart of the system is shown in figure 8 . Initial point will be the implementation of realtime measurements.

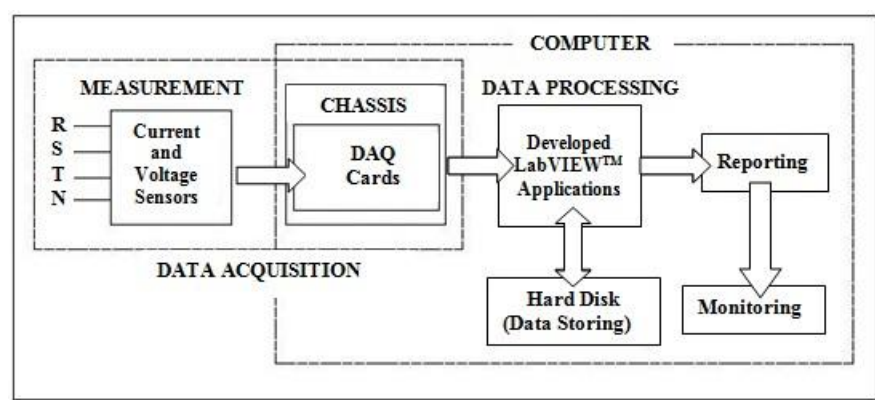

Fig. 8. Flow chart of the expert system.

Developed measurement system is composed of 3 voltage sensors (LEM LV 25P) and 3 hall-effect current sensors (LEM LTS 25NP). Voltages for each phase are measured at the interface point of the system equivalent impedance and the disturbing load. Currents of the disturbing load are again measured simultaneously for each phase by using the hall-effect current sensors.

Measured data is transferred to the computer for the use of developed LabVIEWTM applications, by the help of DAQ cards and the data acquisition board. NI 9215 having 4 channels of total $400 \mathrm{kSample} / \mathrm{s}$ sampling rate and 16 bits resolution and $\pm 10 \mathrm{~V}$ input range is used together with LEM LTS 25 NP. NI 9203 having 8 channels of total 200 $\mathrm{kSample} / \mathrm{s}$ sampling rate and 16 bits resolution and \pm 20 $\mathrm{mA}$ input range is used together with LEM LV 25P. Both DAQ cards are installed on NI cDaq 9174 chassis which eases the transfer of the data to the computer.

Transferred data related to the measured voltages and currents are evaluated in the developed LabVIEWTM applications. Inside a while loop, FFT is applied to the measured voltages and currents to obtain the harmonic components existing in each frequency (till 50th harmonic).

Voltages' and currents' components are put in a form of array and impedances for each frequency (for each existing components) are calculated from equation 4:

$$
Z_{\text {sy s.(n) }}=\frac{V_{(n)}}{I_{(n)}}
$$

$\mathrm{n}$ refers to the number of harmonic order while, $\mathrm{Z}_{\text {sys.(n) }}$ refers to the equivalent system impedance at nth frequency. $V(n)$ and $I(n)$ refers to the existing harmonic components of voltages and currents respectively.

Existence of resonance and its type is determined by a kind of analogy mechanism. Existence of resonance is determined by the comparison of the phase angles of the calculated equivalent system impedances, by the help of equation 4. While, the type of the resonance is determined by the comparison of the magnitudes of the calculated equivalent system impedances (minimum value at resonant frequency means there exists a series resonance and maximum value at resonant frequency means there exists parallel resonance).

After the determination of the resonance, expert system continues with the analysis of capacitor's operating conditions. This part of the application is based on fuzzy logic.

The preference of fuzzy logic is because of not to name the current situation just as safe and dangerous as classical logic illustrates, but to name it with four different linguistic terms. These four terms are decided as "dangerous", "attention", "tolerable" and "safe".

Fuzzy logic input membership functions are formed benefiting the both standards examined in section IV, according to the "maximum safety principle". Input membership functions for apparent power, voltage and the current is shown in figure 9, figure 10 and figure 11 respectively.

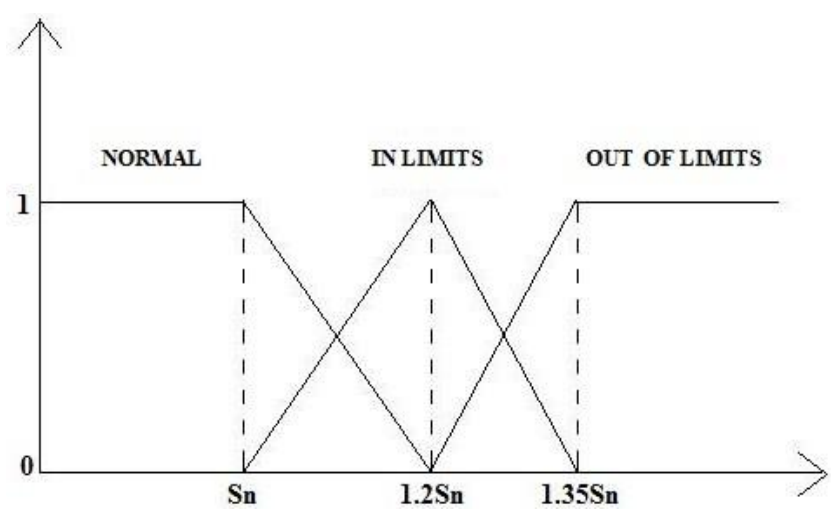

Fig. 9. Input membership functions for apparent power.

Nominal values belonging to the capacitors such as the nominal power, nominal voltage and the capacity value should be defined by the user before the applications start. 


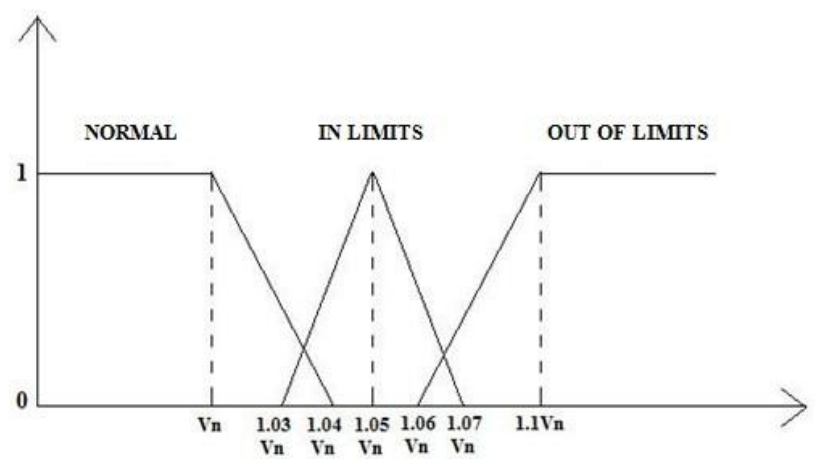

Fig. 10. Input membership functions for voltage.

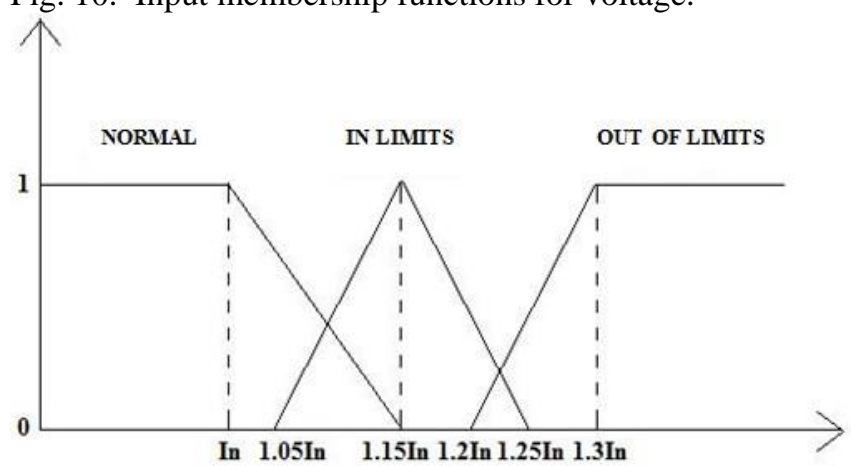

Fig. 11. Input membership functions for current.

As easily seen from the input membership functions, the necessary datas for the decision mechanism are rms voltage, rms current and the apparent power values of the capacitors. Obtained voltage components from the measurements done at the interface point of the disturbing load and the system impedance, are also applied to the ends of the capacitors. In other meaning, for the expert system designed, the only datas collected related to capacitor's operating conditions, belongs to the rms voltages of the capacitors. The best way to get the rms current datas is of course to measure them simultaneously. However, in this study a second way is preferred to optimise the costs. Thus, algorithms are developed to calculate the rms current and the apparent power datas. RMS currents are calculated from the measured and obtained voltage components and the capacity values defined at the beginning by the user. From the capacity values defined, reactance values are calculated for each frequency first:

$$
X_{C n}=\frac{1}{2 \pi f_{n} C}
$$

Then these calculated reactance values form an array and existing current components of the capacitors are calculated from expression 6 at each frequency:

$$
I_{C n}=\frac{V_{C n}}{X_{C n}}
$$

$V_{C n}$ refers to the voltage components from first to 50th harmonic. The true rms value of the current of the capacitors is calculated finally from expression 7 :

$$
I_{C r m s}=\sqrt{I_{1}^{2}+I_{2}^{2}+\ldots \ldots \ldots . .+I_{50}^{2}}
$$

Apparent power is also calculated from the calculated rms current and the rms voltage as shown in expression 8:

$$
S_{C}=V_{r m s} * I_{r m s}
$$

The decision mechanism of determining the capacitor

\begin{tabular}{|c|}
\hline If "V" is out of limits, then $U$ is "DANGEROUS". \\
\hline If "I" is out of limits, then U is "DANGEROUS". \\
\hline If " $\mathrm{S}$ " is out of limits, then $U$ is "DANGEROUS". \\
\hline $\begin{array}{l}\text { If "V" is in limits and "I" is normal and " } \mathrm{S} \text { " is in } \\
\text { limits, then } U \text { is "ATTENTION". }\end{array}$ \\
\hline $\begin{array}{l}\text { If "V" is in limits and "I" is in limits and "S" is } \\
\text { normal, then U is "ATTENTION". }\end{array}$ \\
\hline $\begin{array}{l}\text { If "V" is normal and "I" is in limits and " } \mathrm{S} \text { " is in } \\
\text { limits, then } U \text { is "ATTENTION". }\end{array}$ \\
\hline $\begin{array}{l}\text { If "V" is normal and "I" is normal and " } \mathrm{S} \text { " is in } \\
\text { limits, then } U \text { is "TOLERABLE". }\end{array}$ \\
\hline $\begin{array}{l}\text { If "V" is in limits and "I" is normal and " } \mathrm{S} \text { " is } \\
\text { normal, then } U \text { is "TOLERABLE". }\end{array}$ \\
\hline $\begin{array}{l}\text { If "V" is normal and "I" is in limits and " } \mathrm{S} \text { " is } \\
\text { normal, then } \mathrm{U} \text { is "TOLERABLE". }\end{array}$ \\
\hline $\begin{array}{l}\text { If " } V \text { " is normal and "I" is normal and " } \mathrm{S} \text { " is normal, } \\
\text { then } U \text { is "SAFE". }\end{array}$ \\
\hline
\end{tabular}
safety level is completed with the fuzzy rules given in Table III.

Table III Fuzzy rules

\section{Test and Results}

A three-phase model circuit is designed to test the developed expert system is shown in figure 12 . According to the designated components, a parallel resonance should be observed at $250 \mathrm{~Hz}$ in $\mathrm{R}$ phase and at $350 \mathrm{~Hz}$ at $\mathrm{S}$ phase, while no resonance expected in $\mathrm{T}$ phase.

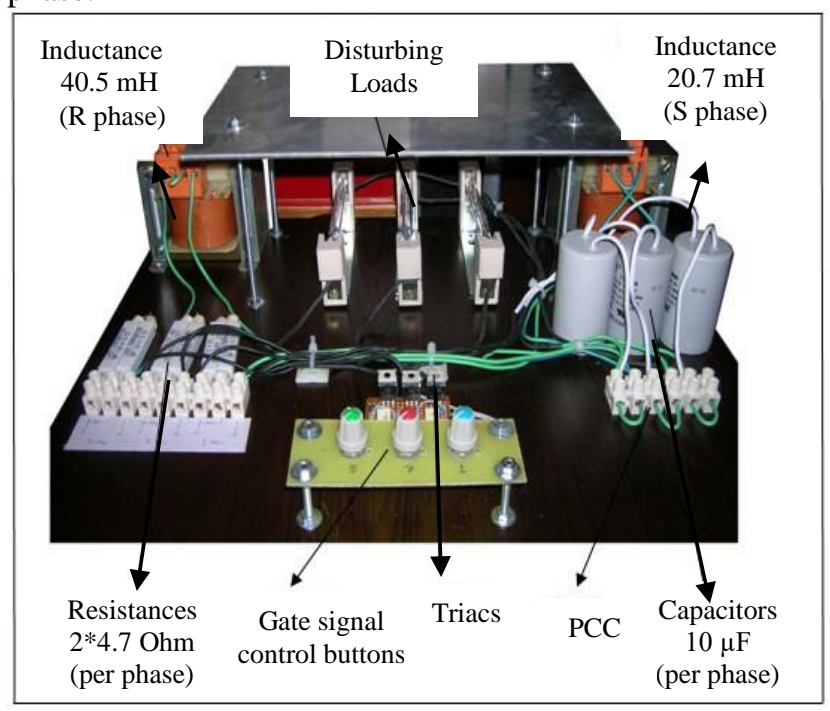

Fig. 12. Three-phase model circuit. 
Three halogen lamps (300 Watts for each phase) are fed through the triacs, representing the disturbing loads. By the help of triacs, the spectral content and the magnitudes of the current harmonics are controlled.

Currents for each phase of the disturbing load and the terminal phase-neutral voltages are measured. Variations obtained in the calculated equivalent system impedances can be seen in figure 13 and figure 14.

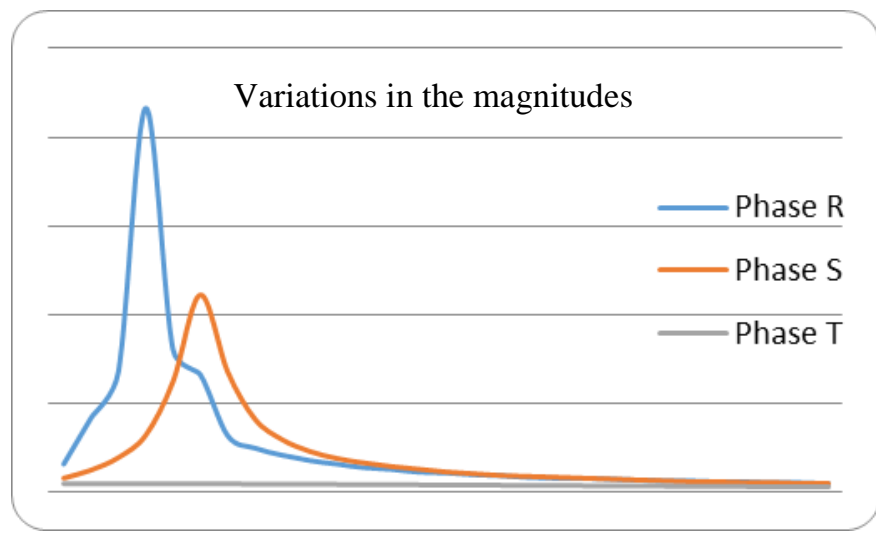

Fig. 13. Test results, variations in the magnitudes of the equivalent system impedances.

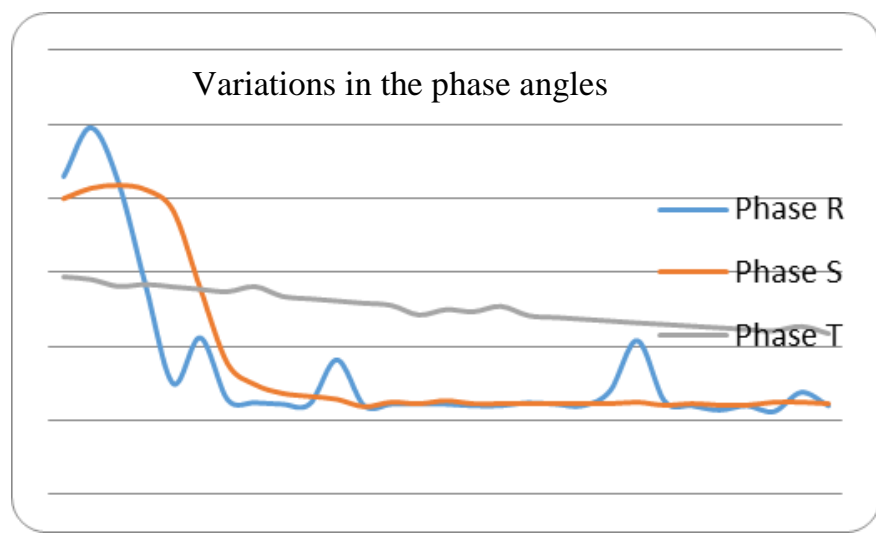

Fig. 14. Test results, variations in the phase angles of the equivalent system impedances.

Finally, the informative output of the expert system is presented in Table IV.

Table IV informative output of the expert system

\begin{tabular}{|l|l|l|}
\hline Phases & $\begin{array}{l}\text { Determination of the } \\
\text { Resonance }\end{array}$ & $\begin{array}{l}\text { Determination of the } \\
\text { Capacitor Safety }\end{array}$ \\
\hline $\mathrm{R}$ & $\begin{array}{l}\text { There is a parallel } \\
\text { resonance at } 250 \mathrm{~Hz} .\end{array}$ & Safe. \\
\hline $\mathrm{S}$ & $\begin{array}{l}\text { There is a parallel } \\
\text { resonance at } 350 \mathrm{~Hz} .\end{array}$ & Safe. \\
\hline $\mathrm{T}$ & - & Safe. \\
\hline
\end{tabular}

\section{Conclusion}

It is emphasized in this study that, determining the existence of a harmonic resonance and its type is feasible in some conditions. With the expert system designed, it is possible to observe the effects of a possible resonance on the capacitors. The part related to the determination of the capacitor safety level may be adapted to any system components or to the loads if desired.

The biggest advantage of the system is to save the researchers from the difficulties of modelling undoubtedly. However, the most important disadvantage is the deviations and wrong results caused by the reversely injected harmonic components and the existence of other distortive signals such as dc components, noises etc.

Another disadvantage may be said as the cost of the system due to the necessity of some measurement and data acquisition components.

\section{Acknowledgement}

The authors are pleased to acknowledge the financial support for this research from Istanbul Technical University, Office of Scientific Researches Projects (ITU-BAP) Project No. 33792.

\section{References}

[1] Guide for Application of Shunt Power Capacitors, IEEE Std. 1036, 1992.

[2] Shunt Power Capacitors of The Self-Healing Type for A.C.Systems Having a Rated Voltage Up To and Including 1000 V; Part 1: General, Performance, Testing and Rating; Safety Requirements; Guide for Installation and Operation, IEC 60831-1, 2002.

[3] M. Nagpal, W. Xu and J. H. Sawada, Harmonic Impedance Measurement Using Three-phase Transients, IEEE Trans. On Power Delivery vol.13 pp.272-277, 1998.

[4] A. S. Morched and P. Kundur, Identification and Modelling of Load Characteristics at High Frequencies, IEEE Trans. on Power Systems vol. 2, no.1, pp. 153-160, 1987.

[5] A. A. Girgis and R. B. McManis, Frequency Domain Techniques for Modelling Distribution or Transmission Networks Using Capacitor Switching Induced Transients, IEEE Trans. on Power Delivery vol. 4 pp. 1882-1890, 1989.

[6] A. Greenwood, Electrical Transients in Power Systems, 2nd Edition, John Wiley\&Sons, 1991.

[7] B. Palethorpe, M. Sumner and D. W. P. Thomas, Power System Impedance Measurement using a Power Electronic Converter, Harmonics and Quality of Power, 2000. Proceedings. Ninth International Conference. Vol. 1 pp.208 -213 , October 2000.

[8] Y. Xiao, J.-C. Maun, H. B. Mahmoud, T. Detroz and S. Do, Harmonic Impedance Measurement Using Voltage and Current Increments from Disturbing Loads, Harmonics and Quality of Power, 2000 Proceedings. Ninth International Conference vol.1, pp. 220-225, 2000.

[9] D. Crevier and A. Mercier, Estimation of Higher Frequency Network Equivalent Impedances by Harmonic Analysis of Natural Waveforms, IEEE Trans. on Power Apparatus and Systems, vol. pas-97, no. 2, pp.424 - 427, 1978.

[10] J. Bird, Electrical Circuit Theory and Technology, Newnes, 2003. 